\title{
Forecasting neu gedacht
}

\section{Liebe Leserinnen und Leser,}

Forecasting war lange Jahre das Aschenputtel der Unternehmenssteuerung. In Lehrbüchern wird der Prozess bis heute im Regelfall nur am Rande thematisiert. Und auch in der Praxis war lange Zeit Unverständnis die typische Reaktion, wenn man sich ausgerechnet mit diesem Prozess intensiver beschäftigen wollte. Letzteres hat sich nun spürbar geändert. Zum einen wird die scheinbar selbstverständliche Verknüpfung des Forecastings mit dem Planungsprozess zunehmend hinterfragt, und zum anderen schafft der Einsatz von Predictive Analytics ein zweites Element mit dem Potenzial für radikale Veränderungen der Forecasting-Praxis.

Zunächst zum ersten Aspekt: Das Junctim zwischen Forecasting und Zielsetzung wurde - vor gut 15 Jahren - zum ersten Mal von Jeremy Hope und Robin Fraser problematisiert. Die beiden stellten eine ganz einfache Frage: Wie kann ein Forecast unsere bestmögliche Einschätzung der zukünftigen Entwicklung wiedergeben, wenn er in den Planungsprozess einfließt und daher - gewissermaßen durch die Hintertür auch die Zielsetzung beeinflusst? Die Konklusion der beiden war so einfach wie naheliegend: Er kann es nicht. Lasst uns daher das Vielzweckprodukt Budgetierung entbündeln und die auf Forecasts aufbauende Ableitung absoluter Zielvorgaben durch relative Bewertungen in Form von wettbewerbsorientierten Benchmarks ersetzen. Im Ergebnis - so der Gedankengang - werden sowohl die bestmögliche Einschätzung der Zukunft im Rahmen des Forecasts als auch die Ableitung von Zielen nicht mehr durch politische Überlegungen beeinflusst.

Der zweite Aspekt mit hohem Veränderungspotenzial verbirgt sich hinter dem populären Begriff der Predictive Analytics. Was steckt dahinter? Große Mengen interner und externer Daten werden mithilfe eines Algorithmus dazu genutzt, Forecasts zu generieren. Die damit verbundene Automatisierung des Prozesses verspricht enorme Effizienzpotenziale. Sie kann zudem dabei helfen, den Forecast weiter zu objektivieren - systematische Verzerrungen durch individuelle Biases oder politisches Verhalten werden durch den Einsatz von

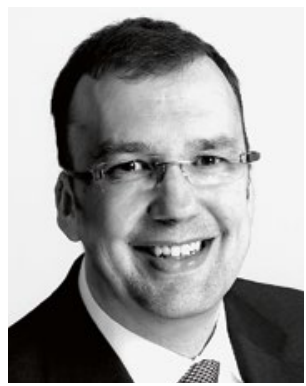

Utz Schäffer

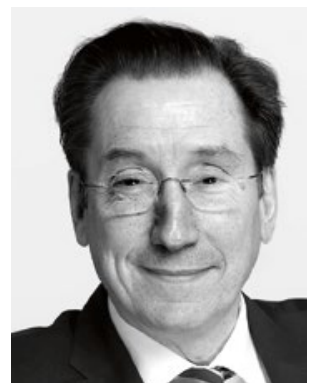

Jürgen Weber
Predictive Analytics an den Rand gedrängt. Und die Liste der Vorteile hört an dieser Stelle nicht auf: Ein automatisierter Prozess kann auch so gestaltet werden, dass beim Verlassen eines vorab definierten Korridors erlaubter Zukunftswerte Gegenmaßnahmen angestoßen werden. Der Einsatz von Bandbreiten erhöht so die Reaktionsfähigkeit des Unternehmens, und die Kalenderorientierung des Prozesses gehört der Vergangenheit an. Trotz dieses enormen Potenzials ist aber auch Vorsicht geboten: Predictive Analytics können nur so gut sein wie die zugrunde liegenden Daten, und auch bei Strukturbrüchen stößt der Ansatz schnell an Grenzen.

Fassen wir zusammen: Der Gedanke des Unbundlings und Predictive Analytics stellen beide die herrschende Forecasting-Praxis ganz fundamental infrage. Die Chancen sind groß, das Potenzial für Fehler aber auch. Grund genug, intensiv in dieses Thema einzusteigen.

Viel Vergnügen bei der Lektüre wünschen Ihnen

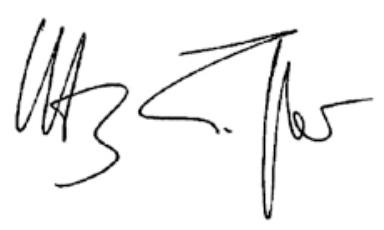

Utz Schäffer

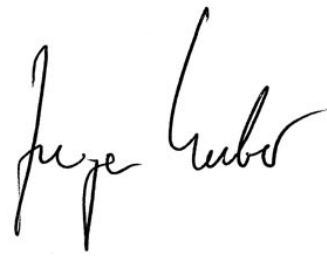

Jürgen Weber 